\title{
A CASE OF RIGHT AORTIC ARCH AND ASSO- CTATED VENOUS ANOMALIES
}

\author{
RUSSELL T. WOODBURAE \\ Department of Anatomy, University of Michigan Medical School, Ann Arbor \\ THRFE FIGURES

\section{INTRODUCTION}

Anomalies of the great vessels about the heart have shared with congenital malformations of the heart in a marked increase of interest in recent years especially with respect to clinical diagnosis and surgical correction. The embryological evaluation of most of these variants has also received the attention of investigators (Poynter, '16; Congdon, '22; Barry, '51) and recently in the more clinically directed articles of Taussig ('47), and Fdwards ('48). The object of the present paper is to place on record the description of a case exhibiting a number of great vessel anomalies in the same individual, the rarity of the case being largely due to the combination of a number of such variants.

\section{DESCRIPTION OF MATERIAL}

The specimen is that of a 66 -year-old male who showed, in the course of routine dissection, no unusual vascular or visceral anomalies elsewhere in the body. At the beginning of the thoracic exposure, however, it was evident that there were aberrancies in both the venous and arterial stems and the body was immediately taken out of student hands and a careful dissection of the chest instituted. It proved to exhibit (figs. 1-3) a right aortic areh of the simpler and more common type, a right descending (thoracic) aorta, the persistence of a left superior vena cava, bilaterally symmetrical azygos veins and a completely left-sided thoracic duct. 
The heart is of normal size and appearance and the relation of the aortic and pulmonary trunks unremarkable. The ascending aorta gives place to an arch which shows no tendency to turn toward the left but curves posteriorly on the

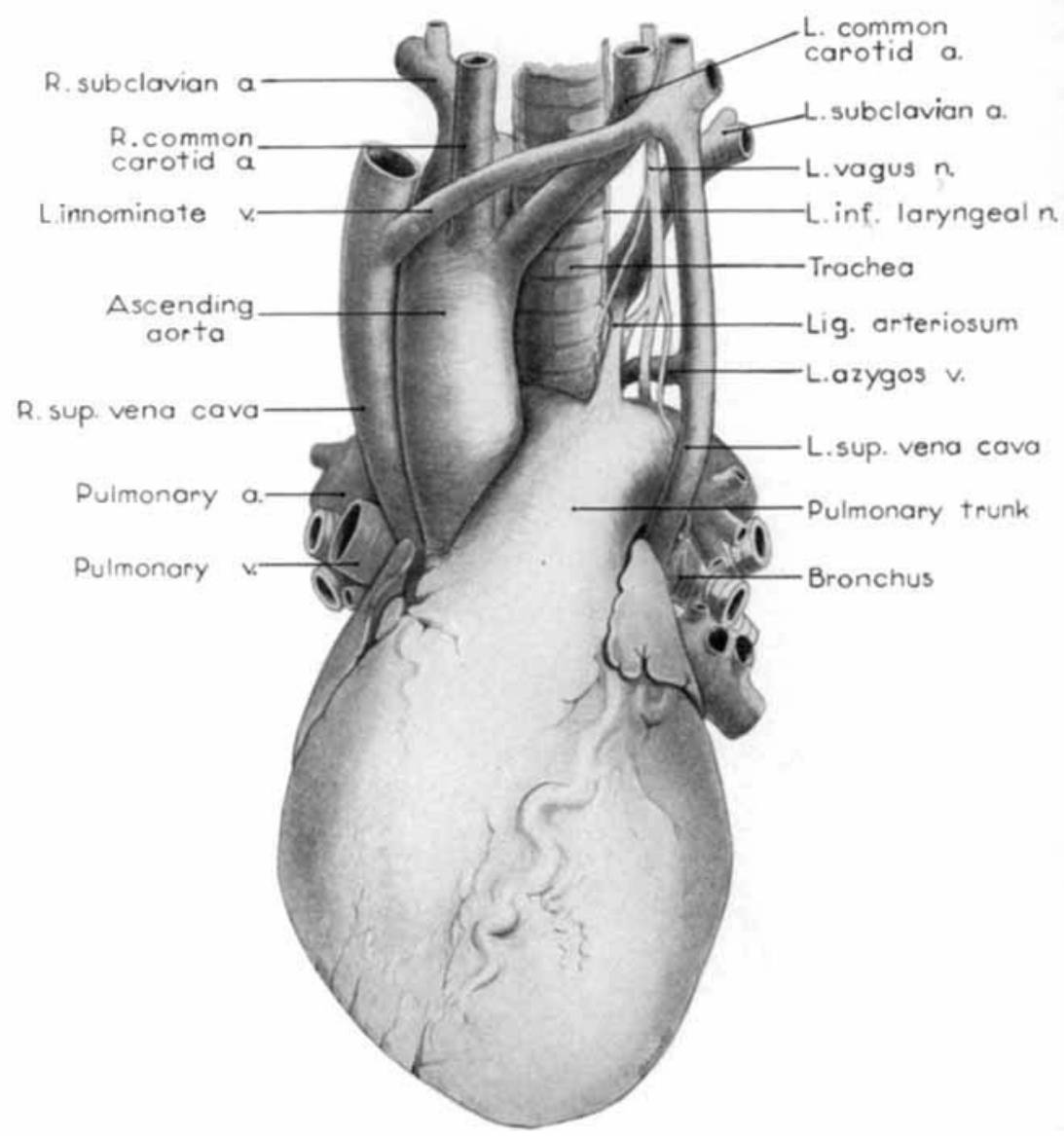

Fig. 1 Ventral view of heart and great vessels.

right of the trachea and esophagus, reaching its summit at the level of the body of the second thoracic vertebra. The arch makes a very sharp turn into the thoracic aorta and as such descends on the right of the vertebral column. The descending aorta remains entirely on the right of the midline 
down to the 10th thoracie level where it curves over on to the midline and then passes through the diaphragm to the left of the midline. After removal of the heart and great vessels it was apparent that there was malformation of eertain tho-

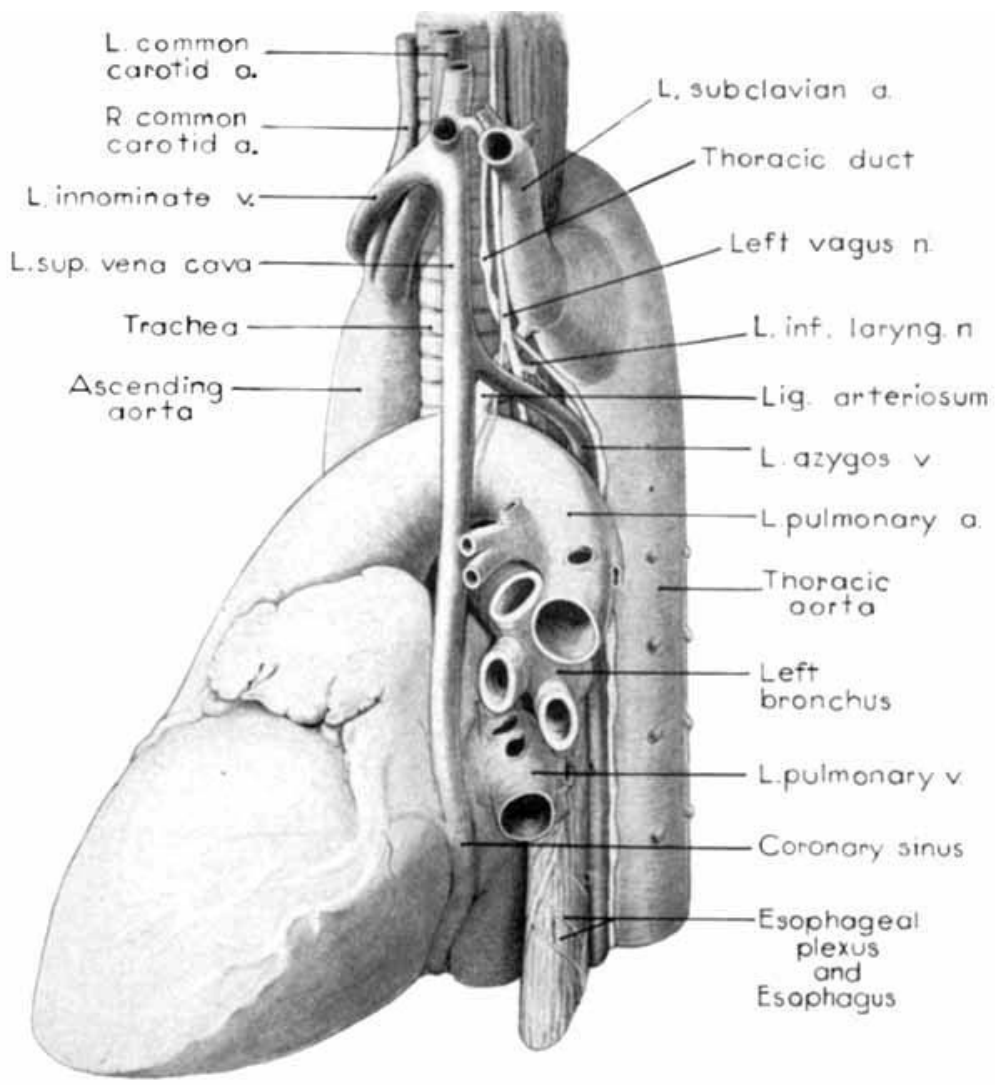

Fig. 2 Left lateral view of heart showing particularly the origin of the left subclavian artery from the aortic diverticulum and the persistent left superior vena cava.

racic vertebrae. Thoracic vertebrae 4,5 , and 6 were eroded, channeled, and flattened on the right side under the proximal portion of the right descending aorta.

The major branches of the arch (figs. 1, 2, 3) are, in the order of emergence, left common carotid, right common caro- 
tid, right subclavian, left subclavian. The left and right common carotids arise adjacent to one another from the first half-inch of the arch, the left one first in order and more to the left side. The left common carotid crosses the trachea

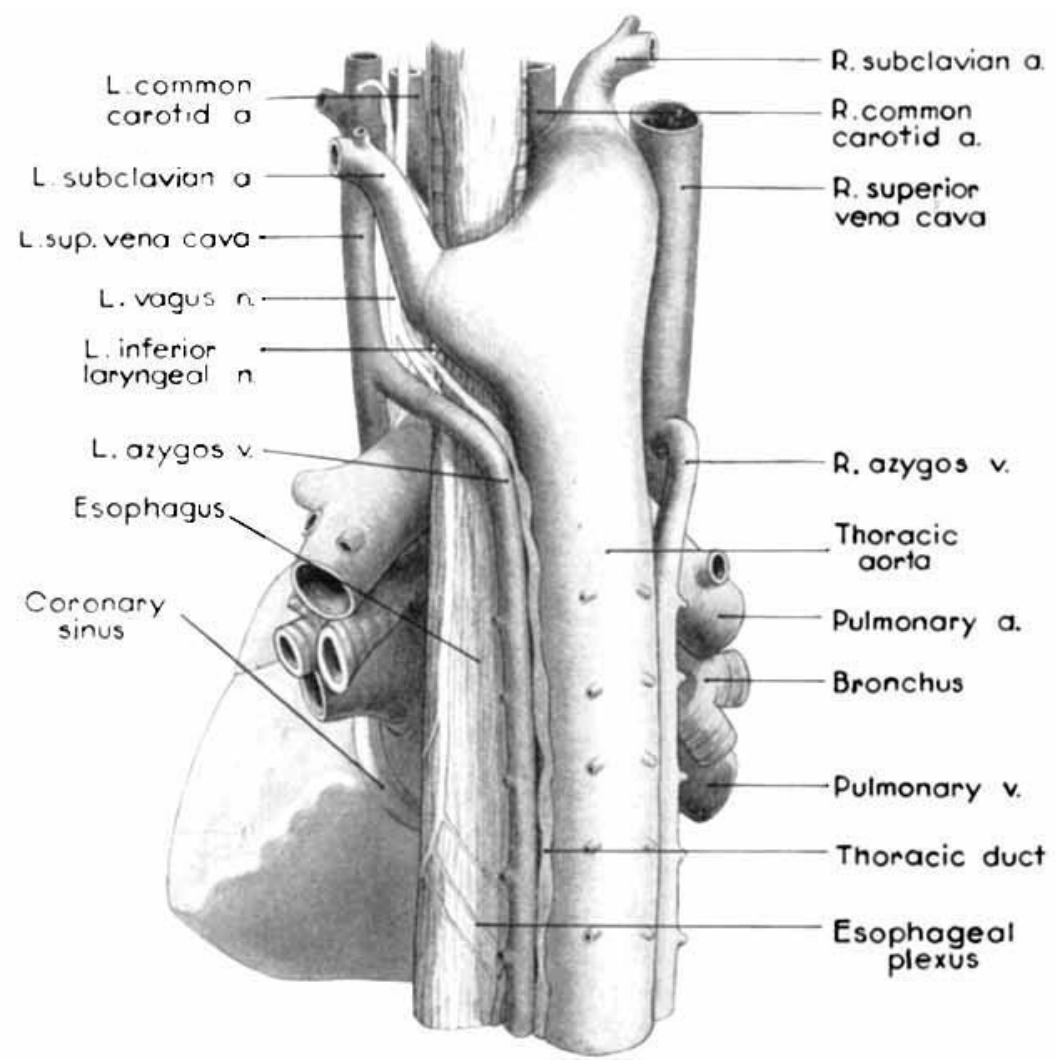

Fig. 3 Dorsal view of the great vessels showing particulariy the aortic divertieulum, the bilateral superior venae eavac, the paired azygos veins and the left thoracie duct.

diagonally to assume its normal relations in the neck. The two common carotids arise immediately adjacent to one another but do not form a common stem as in the case reported by Annan ('10). The right subclavian artery (fig. 3) arises from the very summit of the aortic arch. The left subclavian artery arises from a conical expansion or diverticulum of 
the descending aorta (figs. 2, 3) which passes leftward behind the esophagus between it and the vertebral column. This diverticulum, measuring $25 \mathrm{~mm}$ in length and $25 \mathrm{~mm}$ at its greatest breadth, receives the upper end of the ligamentum arteriosum (figs 1,2) and clearly represents the persistent left root of the dorsal aorta as noted by Turner (1862) and Thomson (1863). Clinical writers (Taussig, Edwards) appcar to consider the more commonly encountered configuration to be a right aortic arch, and a retroesophageal descending aorta which swings rapidly to the midline at about the 4th thoracic level to give rise to the left subclavian artery, which in turn receives the upper end of a left ligamentum arteriosum. Such a pattern corresponds to the case deseribed by Priman ('29). Whether the aorta itself or a diverticulum of it passes retroesophageally to give rise to the left subclavian artery the conditions tending toward compression of trachea and especially of esophagus are essentially the same.

The ligamentum arteriosum in this case (figs. 1, 2) extends from the root of the left pulmonary artery diagonally upward, backward, and to the left to terminate at the under side of the extremity of the left aortic diverticulum. It is somewhat longer than usual, measuring $25 \mathrm{~mm}$ in length and $5 \mathrm{~mm}$ in its greatest transverse diameter. There was nothing unusual concerning the further course or branches of the major vessels. The vertebral arteries entered the costotransverse foramina of the 6 th cervical vertebrac, indicating that the subclavian arteries have the usual origin from the 7 th dorsal intersegmental arteries (Barry, '51).

The nerve relations are those to be expected with this aortic anomaly. The right vagus passes the arch of the aorta at the highest point of its curve and sends its recurrent or inferior laryngeal branch under the abrupt bend of the arch, which is opposite the origin of the right subclavian artery. The left vagus (figs. 2, 3) descends across the left subclavian artery, lies in contact with the left aortic diverticulum and sends its recurrent laryngeal branch under the upper end of the ligamentum arteriosum in the usual relation to that 
structure. Both vagal nerves continue downward to normal relationships with the bronchi and esophagus.

The associated venous abnormality is the presence of a left superior vena cava (figs. 1-3). This represents a persistence on the left of the anterior cardinal and common eardinal veins and the proximal portion of the posterior cardinal vein. The left superior cava begins with the union of the left subclavian and internal jugular veins and descends to penetrate the pericardium just below the left pulmonary artery. On the heart it lies (fig. 2) on the dorsal wall of the left atrium just to the left of the origin of the left pulmonary veins, in the typical position of the oblique vein of the left atrium. Thence it continues to enter the left end of the coronary sinus (figs. 2,3 ). In its extrapericardial course it receives a left azygos vein which arches across the left pulmonary artery to join it. The left superior vena cava in this specimen is $7-8 \mathrm{~mm}$ in transverse diameter, distinctly smaller than the normal right superior vena cava, and united with it by means of a somewhat smaller than normal left innominate vein (fig. 1).

Bilaterally paired azygos veins (figs. 1-3) are present in this specimen. Posteriorly in the chest they parallel and lie against the thoracic aorta, one to each side. 'They are devoid of any "cross connection through thoracic levels and each arches forward over the pulmonary root to enter the superior vena cava of its side at the level of the 4 th thoracic vertebra. Fach, too, receives the superior intercostal vein as it turns forward over the pulmonary root. The right azygos vein is the larger of the two.

The thoracic duct (figs. 2, 3) has an interesting relationship in the specimen under discussion. It aseends from the level of the diaphragm completely to the left of the midline in the interval between the aorta and the left azygos (normally hemiazygos) vein, a reversal of its usual relation to aorta and azygos vein. As the left azygos vein arches forward over the pulmonary root, the thoracic duct continues upward ventral to the left aortic diverticulum (fig. 2), between 
the left vagus nerve and the left subclavian artery, and finally anteriorly to empty in a normal manner at the junction of the left subclavian and left internal jugular veins. There was no indication of a right lymphatic trunk in the chest.

The esophagus shows an adaptation to the right descending aorta. In the neck it was found distinctly to the right of the midline. It deviates to the left at aortie arch levels and is considerably compressed between the left aortic diverticulum and the trachea (figs. 2, 3). Escaping below the diverticulum it is displaced further to the left and shows relatively little contact with the vertebral column in the lower thorax. The right arch and its branches, the retroesophageal diverticulum and the ligamentum arteriosum together constitute the vascular and ligamentous ring of dysphagia Insoria.

\section{DISCUSSION}

The various vascular irregularities illustrated by this specimen are readily understood on the basis of embryological development. The right aortic arch represents a retention of the right rather than the left of the arch primordia while the left aortic diverticulum appears to be a persistence of the left dorsal aortic root in the area between the fusion of the primitive roots on the one hand and the 6th arch (becoming distally the ligamentum arteriosum) on the other hand. The pattern exhibited may be theoretically explained by the loss of the left 4 th aortic arch early in development. The bilateral character of the superior venae cavae is the result of a persistence on both sides of the anterior and common cardinal venous channels. The inequality in size of the two sides and the presence of a reduced left innominate vein affords evidence that there was some progress toward the normal configuration before the vessels became fixed in the pattern exhibited. The paired azygos veins of the specimen exhibited a persistence of the bilateral embryonic condition. The erosion and channeling of the midthoracic vertebrae by the thoracic aorta may be considered as the ond result of 
compression factors which prohibited the development of the usual cross anastomoses between the right and left supracardinal systems and thus forced the retention of a left azygos system.

In a report of three cases of right aortic arch Reid ('14) called attention to the presence in certain cases of the thoracie duct to the left of the descending aorta. It is a distinct temptation in the present case to consider the totally left thoracic duct lying between the left azygos vein and the aorta to be the result of suppression of the primordial right channel by the presence of the descending aorta on that side.

While vaseular anomalies of the great vessels are relatively rare, they are sufficiently common to have been studied and recorded in numerous reports in the literature and it is doubtless true that the observed but unrecorded instances are even more numerous. In his comprehensive discussion of aortic arch and branch anomalies, Poynter ('16) listed references covering 56 cases up to 1916 of right arches with the series of major branches in the order of the specimen here described. Sprong and Cutler ('30) found 26 cases in their less complete review of the literature of which 6 cases had been reported later than the publication of Poynter's study. Several other published reports prior to 1930 have come to the attention of the writer, such as Gruber ('12), Mutel and Fourche ('23), and Priman ('29). Thus the above figures give no reliable indication of the occurrence of this vascular anomaly but do serve to indicate that it is at least relatively uncommon.

The occurrence of double superior venae cavae is much more common; indeed every anatomical laboratory has numerous museum specimens of this variation. However, the combined anomaly of right aortic arch and double superior venae cavae appears to be of genuinely rare occurrence. Sprong and Cutler ('30) found the association in only two cases in their search of the literature (Mutel and Fourche, '20, and Hurley and Coates, '27), and Hurley and Coates were aware of no previously reported case. There are doubtless others but the likelihood of combined arterial and venous 
anomalies in the great vessels of such magnitude as these is very small. It is interesting to note certain differences in even these three cases. In that described by Mutel and Fourche ('20) a similar aortic diverticulum exists and is associated with both the ligamentum arteriosum and the left subclavian artery but the descending aorta is described in a median position. In the case of Hurley and Coates ('27) there was no left aortic diverticulum; the aorta swinging toward the midline gave origin to the left subclavian artery at the upper end of the thoracic portion and continued as a normal leftsided thoracic aorta. The ligamentum arteriosum attached to the left pulmonary artery and the left subelavian artery.

The superior venae cavae reported by Mutel and Fourche ('20) were equal in size and were not connected by a left innominate vein. Two azygos veins were present. The left superior vena cava of the case reported by Hurley and Coates ('27) was equal in size to the right cava and was connected to it by a fibrous cord. No mention is made in this report of either azygos veins or thoracic duct. In the case reported here the left superior vena cava was smaller than the right vein. Bilateral azygos veins were related to the two venae cavae.

The cases reported by Harris and Whitney ('27) and Siekert ('49) have certain similarities to each other and to the above discussed three specimens. Fach has a right aortic arch, though differing in its branches, and each exhibits a complete arterial ring around trachea and esophagus. The left side of the ring is formed by a patent ductus arteriosus and patent left dorsal aortic root in the case of Siekert and by means of a left dorsal aortic root confluent with a left innominate artery from the right arch in the case of Harris and Whitney. In the latter case a left superior vena cava was present but drained only the left subclavian vein, the left internal jugular vein crossing to the right caval system. Bilateral azygos veins occurred in this specimen. A small patent channel, having the relations of a left superior vena cava, united the coronary sinus with the left superior intercostal 
and left hemiazygos veins in the specimen described by Siekert ('49). It did not drain the internal jugular' and subclavian veins of the loft side.

\section{SUMMARY}

1. A specimen is deseribed in which a right aortic areh gives rise successively to left common carotid, right common carotid, and right subclavian arteries.

2. An aortic diverticulum, representing the left dorsal aortic root in development, passes to the left bchind the esophagus, gives origin to the left subclavian artery and is connected to the upper extremity of the ligamentum arteriosum.

3. A left superior vena cava is present draining into the coronary sinus. It is connected to the normal larger right superior vena cava by a moderate-sized left innominate vein.

4. Bilateral azygos veins, unconnected in the chest, arch over the pulmonary roots to empty into the superior venae cavae.

5. The thoracic duct has a left-sided thoracic course lying between the aorta and the left azygos vein.

6. The esophagus is markedly deviated in its course by the aberrant blood vessels and is considerably constricted by the aortic diverticulum behind it.

7. Brief consideration is given to the embryological background of these anomalies and to similar cases reported in the literature. The particular combination of major anomalies exhibited here has come to the writer's attention in only two previously reported cases.

\section{LITERATURE CITED}

A.Nan, J. L. 1910 Case of abnormal sinuous aorta. J. Anat. and Physiol, 44: $241-243$.

BARRY, A. 1951 The aortic arch derivatives in the human adult. Anat. Rec., 111: 221-238.

Congdon, E. D. 1922 Transformation of the aortic arch system during the development of the human embryo. Contrib. to Eubryol., 14: 47-110. Carnegie Contrib. to Embryol. 
Enwards, J. E. 1948 Anomalies of the derivatives of the aortie areh system. The Medical Clinies of North America, Mayo Clinic Number, 925-949.

Gruber, G. B. 1912 Zwei Fälle von Dextropositio des Aortenbogens. Frankfurt Ztschr. f. Path., 10:375-382.

HAREIS, H. A., AND C. WHITAKY 1927 The heart of a child aged nineteen months presenting right and left aortic arehes, with multiple anomalies of the great vessels. Anat. Rec., 34: 221-232.

Hurley, L. E., AND A. E. CoATEs 1927 A case of right-sided aortic areh and persistent left superior vena cava. J. Anat., 61: 333-339.

MUTh: And Fourche 1920 Anomalie complexe artérielle et veineuse dos gros vaisseaux de la base du cocur. Soc. Anat. de Paris, Bull. et Mem., 17: 669-674.

1923 Un cas d'inversion de la crosse aortique. Soc. Anat. de Paris, Bull. at mem., 20: 234-238.

Poynter, C. W. M. 1916 Arterial anomalies pertaining to the aortie arches and the branches arising from then. University Studies of the University of Nebraska, Lincoln, 16:229-345.

Priman, J. 1929 Notes on the anomalies of the aortic arch and of its large branches. Anat. Rec, 42: 335-353.

REm, D. G. 1914 Three examples of a right aortic arch. J. Anat. and Physiol, 48: 174-181.

Siekfr, R. G. 1949 An anomalous human heart. Anat. Ree, 108: 701-709.

Sprong, D. H., JR., AND N. L. CUTler 1930 A ease of human right aorta. Auat. Rec., 45: 365-375.

TAussig, H. B. 1947 Congenital malformations of the heart. N.Y. The Commonwealth Fund. $618 \mathrm{pp}$.

Thomson, A. 1863 Deseription of the dissection of a case of right aortic arch; with remarks on this and allied malformations. Glasgow Med. J, 11: $1-16$.

Tlrner, W. 1862 On irregularities of the pulmonary artery, areh of the aorta, and the primary branches of the aorta, with an attempt to illustrate their mode of origin by a reference to development. Brit. and For. Medico-chirurgieal Rev., 30: 173-189, 461-482. 